\title{
FUNDUS APPEARANCES IN A CASE OF ACUTE DERMATOMYOSITIS*
}

\author{
BY \\ STEWART MUNRO \\ London
}

AlthOUGH dermatomyositis has a vast literature, only four cases showing fundus complications have been reported since their initial description in three patients by Bruce (1938).

This case is presented not only because of the rarity of reports of fundus changes, but also because it differs in some respects from those previously described both in the initial fundus appearances and in their later changes.

O'Leary and Waisman (1940) reported forty cases, and finding no fundus changes in thirty of these, assumed it to be a rare complication. Sheard (1951) analysed 25 cases with no mention of fundus abnormalities. Wedgwood, Cook, and Cohen (1953), in a special review of 26 cases in children tabulated clinical data with no reference to the state of the fundi.

According to Hollenhorst and Henderson (1951), however, the ocular lesions of dermatomyositis, disseminated lupus erythematosus, and serum sickness are "entirely silent in the majority of cases". Walton and Adams (1958) found no fundus changes in any of their forty cases of "polymyositis", but suggested that since the pupils were not dilated before examination the retinopathy may have been overlooked. One may therefore assume that retinopathies in the collagen group of diseases are perhaps less rare than reports suggest.

This is the sixth report of fundus complications since Bruce's original paper, the other communications being those of Lisman (1947), London (1950), Nutt (1951), and de Vries (1951). Successive fundus illustrations have been made of the case forming the subject of the present paper, which differs from those previously described mainly in the retinal arteriolar changes, and, with one exception, in the permanent damage which ensued.

Dermatomyositis, one of the group of collagen diseases, may be acute or chronic in type, affecting striated muscle and involving the skin. There is a gradual onset with vague and indefinite prodromata followed clinically by multiple muscle inflammation oedema, and dermatitis.

The acute form of the disease affects the proximal muscles, is usually accompanied by a skin eruption, and is self-limiting. The chronic, inevitably progressive type, also usually proximal without dermatitis, is characterized by advanced muscle wasting, contractures, tough leathery skin, and severe

\footnotetext{
* Received for publication October 14, 1958. 
joint changes. In these respects, it is (according to Adams, Denny-Brown, and Pearson, 1953) similar to chronic scleroderma. The incidence of acute dermatomyositis is higher in children than that of any other collagen disease, unless rheumatic fever be included, whilst mortality appears to be in the region of 40 to 50 per cent., with the highest incidence between the fourth and fifth decades. Involvement of the respiratory muscles is the main factor in a fatal outcome.

Walton and Adams (1958) classified their forty cases into four groups:

(1) Polymyositis (a) acute with myoglobinuria

(b) subacute or chronic.

(2) With muscular weakness the dominant feature, but with evidence of an associated collagen disease or dermatomyositis with severe muscular disability and minimal or transient skin changes.

(3) Severe collagen disease with minimal muscle weakness, or dermatomyositis with florid skin changes and minor muscular disability.

(4) Dermatomyositis with malignant disease or carcinomatous myopathy.

The Raynaud phenomenon occurred in Groups 2 and 3.

The most common initial symptoms of acute dermatomyositis are muscular weakness and easy fatiguability. A gradual and insidious onset is particularly common, although occasionally an abrupt febrile onset may occur.

The muscles of the shoulder girdle are usually affected at first, soon followed by proximal muscle weakness in the lower extremities. The oedematous lids so characteristic of acute dermatomyositis have been attributed to involvement of the orbicularis oculi. Difficulty in swallowing and speaking indicate that the palatal, pharyngeal, and laryngeal muscles are affected. Exophthalmos, which was described by Heyn (1924), and "tenonitis" could be caused by involvement of the extra-ocular muscles. Dowling and Griffiths (1939) drew attention to a close association between dermatomyositis, thyrotropic myopathy, and myasthenia gravis, in which he considered the muscle changes to be almost identical. Creatinuria he found common to toxic goitre, dermatomyositis, and myasthenia gravis, while the calcium metabolism was disturbed in thyroid disease as well as in dermatomyositis. Weak extra-ocular muscles are found in 10 per cent. of cases according to O'Leary and Waisman (1940). Nystagmus was noted in the first case to be reported by Wagner (1863).

Thoracic muscle weakness and diaphragmatic involvement also occur and may be indirectly the cause of death.

Involvement of the skin of the face occurs early in the disease. Wedgwood, Cook, and Cohen (1953) found this present in 23 out of 26 children in their special review. The characteristic lesion they observed was a violaceous rather than erythematous rash mainly peri-orbital but at times extending to involve the bridge of the nose, malar areas, and upper lip. They suggest that the rash in dermatomyositis is more indurated and less pronounced 
than the typical "butterfly" distribution of systemic lupus erythematosus. Peri-orbital oedema was considered to be the most common ocular lesion by Hollenhorst and Henderson (1951), and O'Leary and Waisman (1940) found this in 25 of the forty cases they reported. The rash may be transitory, affecting not only the face, but spreading rapidly to the trunk and legs. In the case reported here, the pressure areas were affected, requiring careful nursing. Desquamation is common, and petechiae and telangiectasis may be present, perhaps contributing to a "heliotrope" discolouration of the lids. Alopecia is not infrequent. Lesions of the mucosae are occasionally observed, and give rise to stomatitis, pharyngitis, and conjunctivitis. Finally, iritis has been reported by Lisman (1947) and the retinopathy, which in the experience of Hollenhorst and Henderson (1951) is a common ocular complication, was actually seen only in a small percentage of casés.

Pathological changes occurring in the vessels show annular or localized fibrinoid degeneration of the arteriolar walls, particularly in the vessels supplying the muscles. Intimal thickening, with consequent narrowing of the lumen, and occasional thrombus formation occurs. Keil (1940) considered the fundamental changes were in the vasculature with secondary involvement of the muscle parenchyma. Fahr (1921) spoke of a necrotizing arteriolitis, but Talbott and Moleres Ferrandis (1956) state that the characteristic necrotic changes of polyarteritis are not evident in dermatomyositis but that the appearances simulate those of thrombotic thrombocytopenic purpura.

These vessels changes are not considered to be specific for dermatomyositis by Pagel, Woolf, and Asher (1949), and are thought to be absent in the sites of predilection for hypertensive arteriolar changes, e.g. in the kidneys, spleen, and pancreas.

These observers also described muscle changes which were non-specific but occurred in trauma and in muscle subjected to various pathogenic stimuli.

Vacuolation and shrinkage of muscle substance with altered staining affinity and loss of transverse striation is found, while empty sarcolemmellic sheaths filled with macrophages are also present. Early lesions showed extensive oedema of the sub-epithelial connective tissues and muscle bundles, with collections of polymorpho-nuclear leucocytes, mainly eosinophilic. At a later stage, foci of necrosis are surrounded by collections of lymphocytes and monocytes (Wedgwood, Cook, and Cohen, 1953).

\section{Case Report}

A 10-year-old boy was admitted to Guy's Hospital under the care of Dr. R. C. Mac Keith in December, 1957, because of severe muscle weakness complicating a long-standing and puzzling succession of apparently unrelated symptoms.

It was probably after a squint operation in February of that year that an unusual tachycardia which persisted for 3 months following an uncomplicated general anaesthetic was noticed. His health, which had always been good, now gave rise to anxiety, since 
he continually complained of lethargy, sore throats, and bouts of low grade pyrexia, and was easily tired. Since several other boys at his school had similar symptoms, recurrent attacks of influenza were diagnosed, and it was not until later in the year when a succession of transient blotchy rashes appeared, associated with swelling of his face and neck, that the diagnosis was doubted. Severe stomatitis then developed, which was thought to be a mycotic infection progressing to cancrum oris. This new complication coupled with extension of the oedema to his shoulders, arms, and trunk created a new urgency and brought about his admission to Guy's Hospital.

Examination.-He was alert but prostrate, so weak that he was unable to sit up. A dry, scaling erythematous rash with fine telangiectasis and petechiae covered swollen lids and extended over the face in "butterfly" distributions (Fig. 1a).

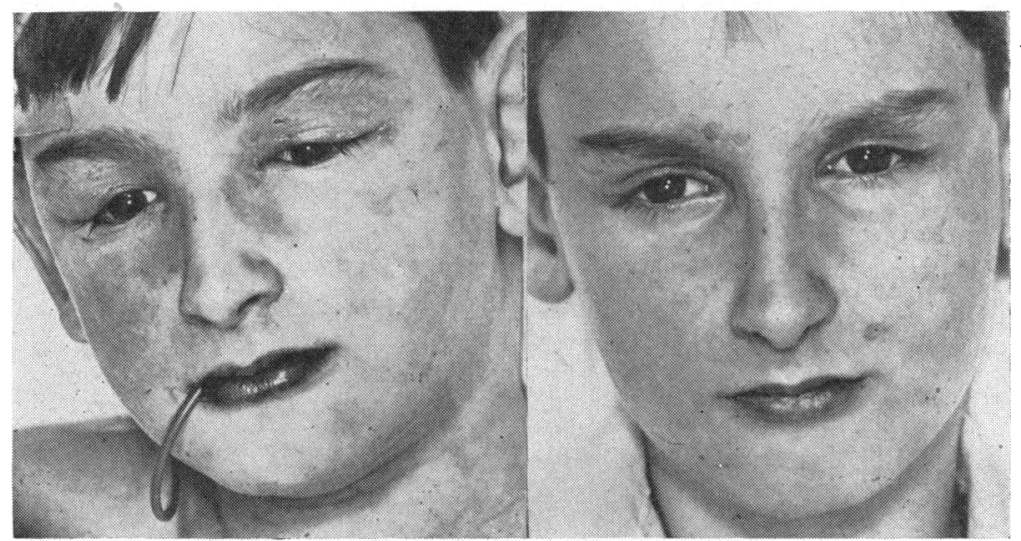

FIG. 1a.-Facies on admission, showing

Fig. 1b.-31 December, 1959. swollen face and scaling erythematous skin condition with "butterfly" distribution (20 December, 1957).

Skin changes over the pressure areas were also present (Fig. 2a), with "pitting" oedema of the shoulders, arms, and trunk (Fig. 3, overleaf).

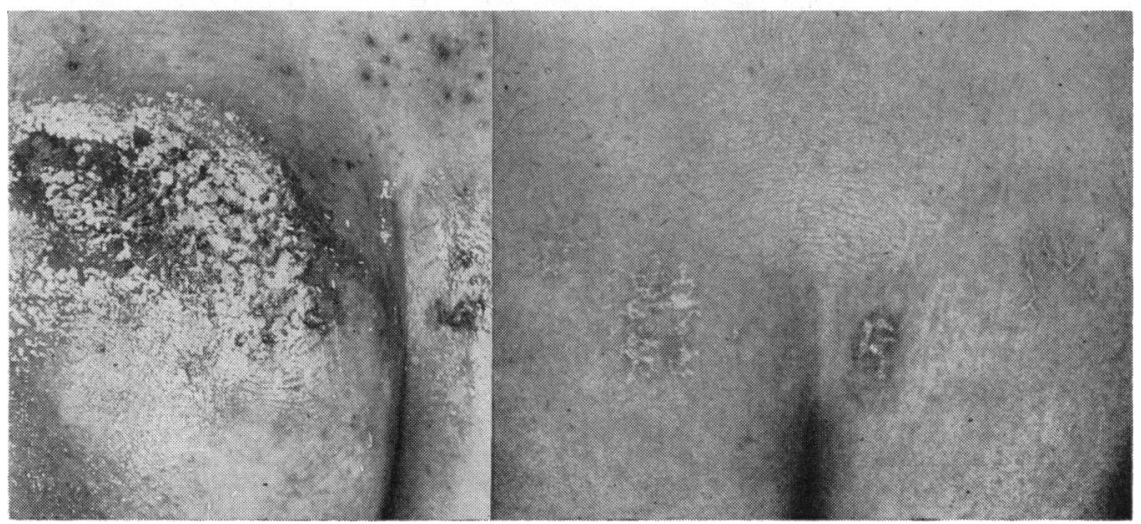

FIG. 2.-Skin lesions of buttocks.

(a) 20 December, 1957.

(b) 31 December, 1957. 


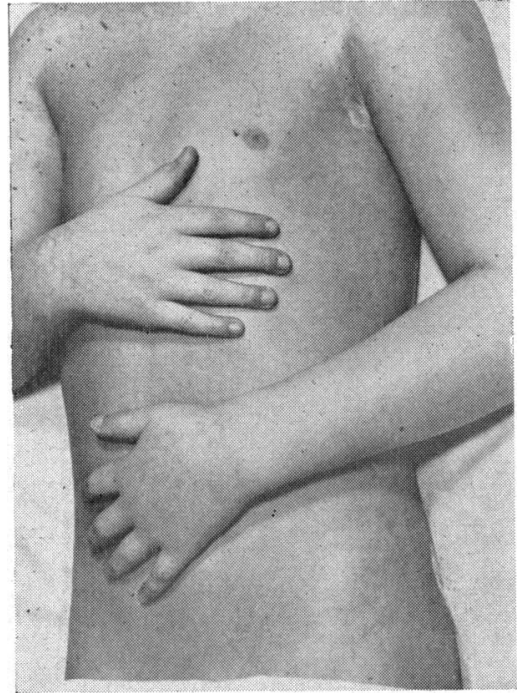

Fig. 3.-Oedema of arms and trunk (20 December, 1957).
The muscle weakness was greatest in the proximal muscles of the arms, the tendon reflexes all being absent. He also had a complete palatal paralysis. External ocular examination revealed no conjunctivitis or anterior uveitis, and the mediae were clear. The eye movements were full and there was no proptosis. The visual acuity, however, was only counting fingers in each eye, whereas it had been $6 / 9$ in the right eye and $6 / 18$ in the left with glasses in February of that year. Fundus examination revealed massive white exudates in both eyes, not only confluent in the macular regions to produce raised masses, but also scattered around the discs, being confined entirely to the posterior poles. The discs were normal, but the retinal arterioles showed marked attenuation with scattered "flame-shaped" haemorrhages along the superior temporal vessels and some scanty deeper haemorrhages. The veins were engorged and the retinae oedematous (Fig. 4).
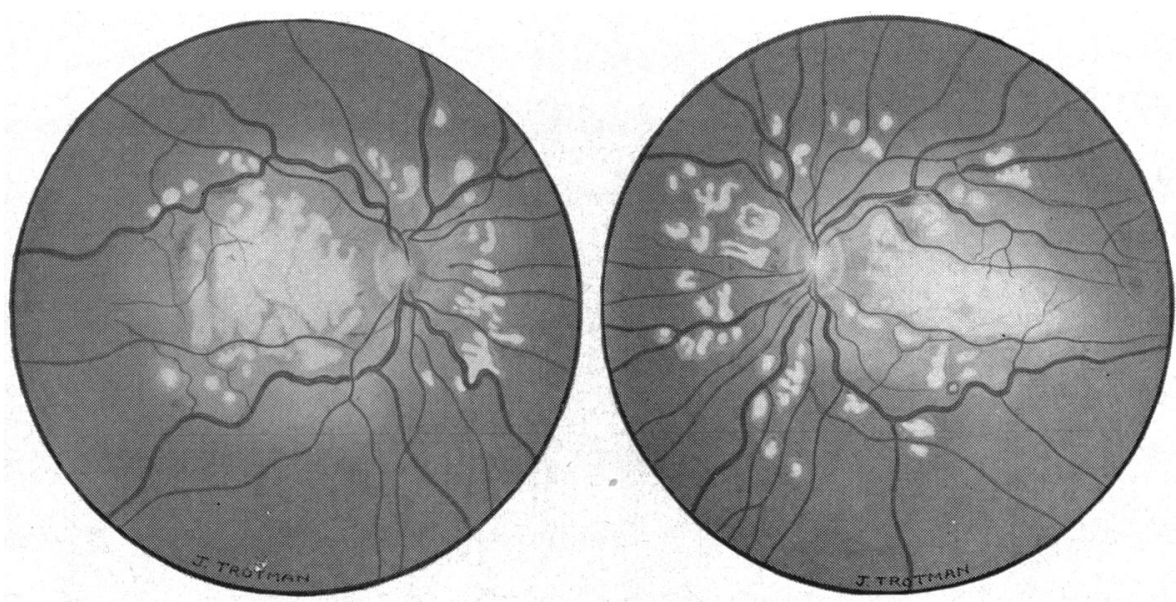

FIG. 4.-Fundus appearances (23 December, 1957).

Routine investigations at this stage showed a slight leucocytosis, a normal erythrocyte sedimentation rate, and a slight trace of albumen in the urine. Apart from a slight sinus tachycardia an electrocardiogram was normal. The blood pressure was raised to 150/120.

Diagnosis.-Dermatomyositis was diagnosed, and because thoracic and diaphragmatic muscle involvement made breathing difficult he was transferred to the National Hospital, Queen Square, where there is a unit for the treatment of respiratory paralysis by intermittent positive pressure. 
Treatment.-Prednisolone therapy was begun, tube feeding and hourly turning on a "ripple" mattress being continued.

Laboratory Investigations. - The main abnormalities revealed were a reversal of the albuminglobulin ratio and a considerable creatinuria.

Serum proteins $6 \cdot 3 \mathrm{~g}$. per cent.

Albumin $2 \cdot 2 \mathrm{~g}$. per cent.

Globulin $4 \cdot 1$ g. per cent.

Urinary creatinine $460 \mathrm{mg} . / 24 \mathrm{hrs}$.

Urinary creatine $450 \mathrm{mg} . / 24 \mathrm{hrs}$.

Clearance $26.6 \mathrm{ml} . / \mathrm{min}$.

Electrophoretic strip showed a relative increase in the alpha and delta globulins.

Full blood count and differential showed no abnormality.

Disseminated lupus erythematosus phenomenon was not demonstrated.

Plasma electrolytes and cerebrospinal fluid were normal.

Blood urea, creatinine, and creatine were normal.

Erythrocyte sedimentation rate $12 \mathrm{~mm} . / \mathrm{hr}$.

Throat swab showed Strept. viridans and saprophytic neisseria.

$X$-ray examination of the chest revealed a slight consolidation in the right upper zone.

Progress.-There was a gradual improvement after treatment. The tachycardia disappeared, blood pressure fell to $130 / 90$ within a few days and within a month of admission he was able to sit up with assistance, but alopecia appeared (Fig. 5).

Examination of the fundi (Fig. 6) now revealed that many of the "soft" exudates had been replaced by "hard" exudates, and these were concentrated in the macular areas.
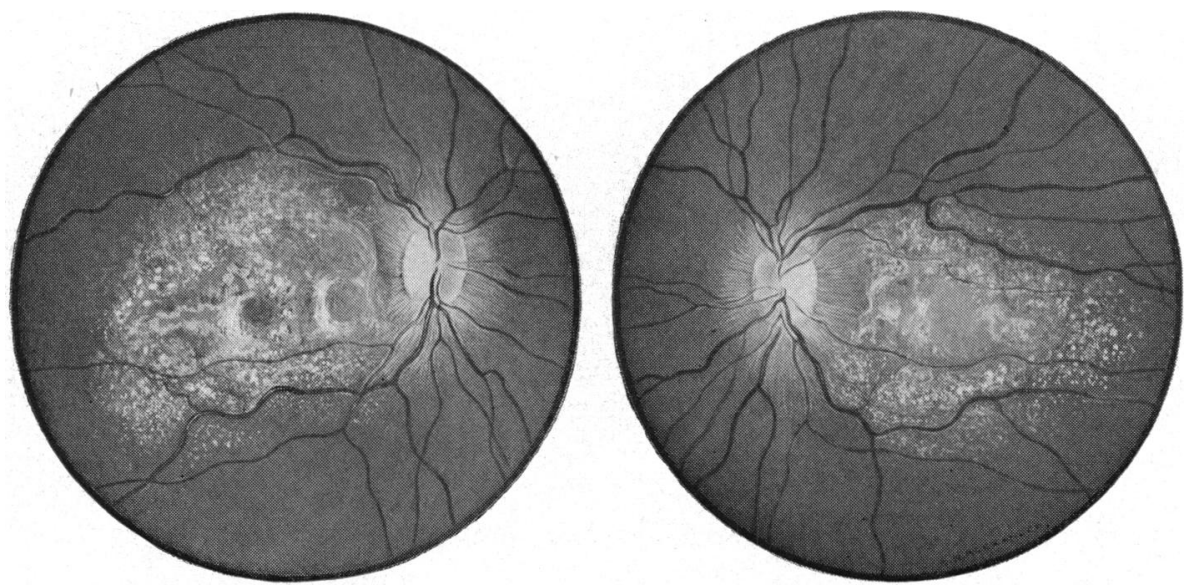

FiG. 6.-Fundus appearances (16 January, 1958). 
The arterioles remained markedly attenuated although the venous engorgement was reduced. The discs now showed temporal pallor and the visual acuity was recorded as $3 / 60$ in the right eye and $1 / 60$ in the left with glasses.

2 months after admission the patient was ambulant and was discharged for convalescence. The fundi (Fig. 7) showed an increase in optic atrophy and hardening with reduction in the number of exudates. The arterioles were still spastic but the veins were now of normal calibre.
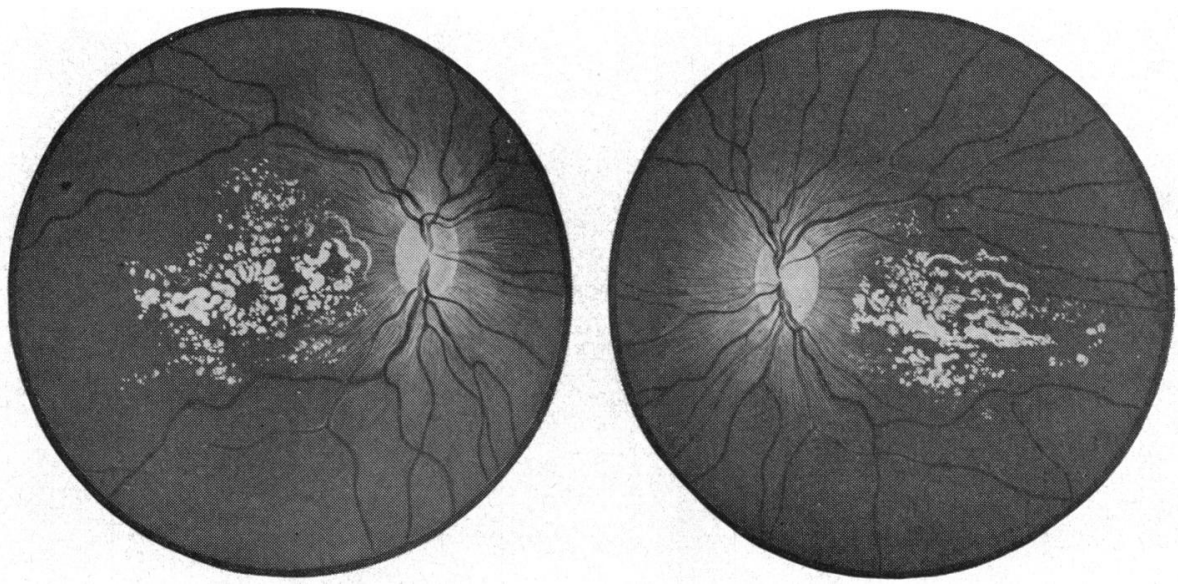

Fig. 7.-Fundus appearances (14 February, 1958).

Result.-When last examined 6 months after onset, he was well and attending school again. Apart from tiredness after walking any reasonable distance and some weakness in abduction of the left shoulder, he had little disability. The fundi at this stage (Fig. 8) showed improvement in the calibre of the arterioles, but the maculae showed pigmentary changes, and there was consecutive optic atrophy.
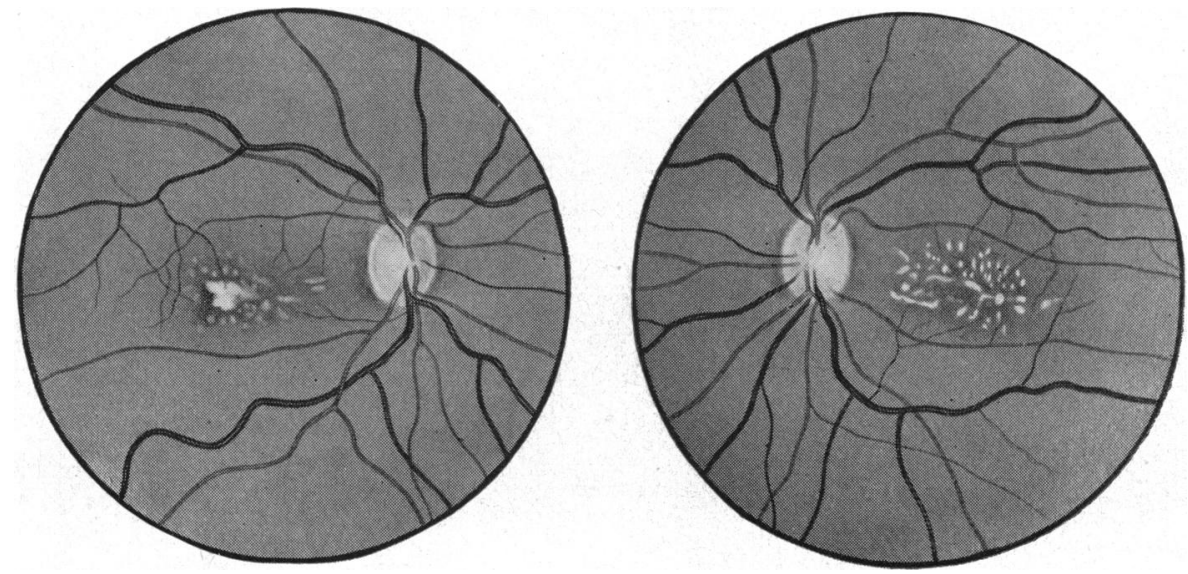

Fig. 8.-Fundus appearances (June, 1958).

The visual acuity was $6 / 60$ in the right eye and 6/60 in the left with glasses $+8 \mathrm{D}$.sph., +3 D.cyl., axis $90^{\circ} \downarrow$ right, and +8 D.sph., +3.5 D.cyl., axis $80^{\circ} \downarrow$ left.

With a visual aid he could read N6 with the right eye and N10 with the left. 


\section{Discussion}

The clinical symptoms and features of this case are typical of the acute form of the disease, but the fundi differ from those previously described in several respects.

Of the three patients reported by Bruce (1938), one of whom died, only one had a slightly raised blood pressure, and all showed normal arterioles with distended veins. Furthermore, after recovery, the fundus appearances returned to normal. The fatal case, however, showed an increasing formation of exudate and haemorrhage.

Pathological examination showed groups of globular bodies in the thickened nerve fibre layer, which were thought to be swollen and varicose nerve fibres. Albuminous deposits occurred mainly in the outer plexiform layers, with a few haemorrhages in the inner plexiform and inner nuclear layers.

Lisman (1947) also reported a fatal case in a man aged 53 in whom the fundus appearances were almost exactly the same as those described by Bruce, but were not at all static. New haemorrhages and exudates appeared as others were absorbed during the short period of observation. Pathological changes were identical and the vessels normal histologically and ophthalmoscopically.

London (1950) described a case in a child of $5 \frac{1}{2}$ years, in which the retinopathy cleared up in 3 weeks after the patient's recovery. The prodromata and clinical features of this case were similar to the case here reported. The blood pressure was slightly raised initially and fell to normal within a few weeks. Fundus examinations showed transient small haemorrhages and white, fluffy exudates which, as in the case described by Lisman, were not static and finally disappeared altogether.

De Vries (1951) described a fatal case in a woman aged 49, whose fundi bore little resemblance to the gross changes in the cases already described. Only a few scattered, soft exudates were present in the posterior poles of the fundi which overlay the vessels in some areas. There were no haemorrhages and the discs and vessels were normal. A pathological report described varicose or ganglion-like swellings in the nerve fibre layer and albuminous deposits separating the parallel nerve fibres. This case had a very high blood sedimentation rate and considerable creatinuria.

Nutt (1951) reported the late fundus changes in a boy of 12 , showing profuse retinal exudate giving the appearance of "snow banking" over the whole of the lower halves of both fundi. The discs and vessels were again normal. In this case residual pigmentary changes affecting the maculae and optic atrophy occurred.

Garcin, Lapresle, Gruner, and Scherrer (1955) remark on the occurrence of floccular retinal exudates and small discrete haemorrhages which may disappear on the patient's recovery. 
It is of interest that the fundus changes reflect the progress of the disease and that an increase of exudation appears during the peak periods of toxaemia. In the present case, however, permanent changes have occurred following the acute phase of the retinopathy, which indicates that the visual prognosis may be poor in contradistinction to that in the patients reported by Bruce (1938) and London (1950), in whom the fundus appearances returned to normal on recovery. No reference to arteriolar involvement has been discovered in the literature of dermatomyositis but is reported to occur in systemic lupus erythematosus where embolic or post-inflammatory arteriolar occlusion may occur and peri-arteritis nodosa when hypertensive retinopathy is seen (Hollenhorst and Henderson, 1951). These writers point out that there is a close association between dermatomyositis and systemic lupus erythematosus and emphasize that the presence of fundus changes is important in differential diagnosis where acute rheumatism or atypical rheumatoid arthritis is suspected. London's case was in fact diagnosed initially as one of rheumatic fever. The initial diagnosis in the 26 children reviewed by Wedgwood, Cook, and Cohen (1953) was correct in only eight, the others being diagnosed as disseminated lupus (four cases), muscular dystrophy (three cases), rheumatic fever (two cases), poliomyelitis (two cases), scleroderma (one case), and so on. The muscular weakness is invariably the primary dysfunction in dermatomyositis, but in systemic lupus erythematosus it is secondary to the systemic manifestations and less pronounced. The rash with the periorbital swelling is usually more violaceous in the former, and involvement of the extra-ocular muscles has not been reported in disseminated lupus erythematosus. Renal involvement is also found frequently in systemic lupus erythematosus but is absent in dermatomyositis.

The fundus appearances in two cases of systemic lupus erythematosus described by Clifton and Greer (1955) are remarkably similar to those found in the present case, differing only in the arteriolar changes, which in their cases consisted only of a slightly heightened reflex without attenuation. The pathological changes were also similar, "cytoid bodies" being present in the distended nerve fibre layer. These "bodies" are the "varicose" swellings previously described. Clifton and Greer (1955) discuss their occurrence and pathogenesis most fully.

I have also seen similar fundus changes in the late stage of a case of scleroderma which before the fatal outcome changed in the course of a few days to bilateral inferior exudative retinal detachments.

The fundus changes of dermatomyositis are therefore not specific to the disease, nor are the pathological changes in the muscles.

Creatinuria also has no specific diagnostic or prognostic value, occurring as it does in extreme functional disorders of skeletal musculature. The albumin-globulin ratio measured in fourteen of the 26 cases reported by Wedgwood, Cook, and Cohen (1953) showed no reversal of the normal 
ratio. Two out of five cases reported by Kinney and Maher (1940) showed reversal of the ratio. Reversal is more common in systemic lupus erythematosus (Talbott and Ferrandis, 1954).

Treatment with systemic ACTH and cortisone has been previously reported by Oppel, Coker, and Milhorat (1950), Sheard (1951), Simpson (1953), Clein (1953), and Lever and Haanes (1954). The last authors point out that there is a more rapid response to cortisone in systemic lupus erythematosus than in dermatomyositis, indicating an important difference in their pathogenesis.

It is of interest that dermatomyositis is not considered to be a rare malady by Talbott and Moleres Ferrandis (1954), who rank it second to systemic lupus erythematosus in incidence among the unusual collagen dyscrasias.

\section{Summary}

(1) A case of dermatomyositis with fundus complications is described.

(2) The unusual feature of this case is the presence of arteriolar spasm.

(3) A close similarity of the fundus lesions to those seen in systemic lupus erythematosus and scleroderma is noted.

(4) The fundus changes are compared with those seen in cases previously reported.

My thanks are due to Dr. M. J. McArdle and Dr. R. C. Mac Keith of Guy's Hospital for permission to publish this case and for kindly allowing me access to their records, and to $\mathrm{Mr}$. Frank Law for his encouragement and advice. I am also indebted to Miss J. Trotman and Mr. E. R. Alexander for their fundus paintings.

Photographs of the external clinical features were provided by the Photographic Department of the National Hospital for Nervous Diseases, Queen Square.

\section{REFERENCES}

Adams, R. D., Denny-Brown, D., and Pearson, C. M. (1953). “Diseases of Muscle”, p. 309. Hoeber, New York.

Bruce, G. M.'(1938). Trans. Amer. ophthal. Soc., 36, 289.

CleIN, L. (1953). Brit. med. J., 2, 1357.

Clifton, F., and Greer, C. (1955). Brit.J. Ophthal., 39, 1.

DE VRIES, S. (1951). A.M.A. Arch. Ophthal., 46, 432.

Dowling, G. B., and GrifFiths, W. J. (1939). Lancet, 1, 1424.

FAHR, T. (1921). Arch. Derm. Syph. (Berl.) [Orig.], 130, 1.

GARCIN, R., LAPresle, J., GrUNER, J., and ScherRer, J. (1955). Rev. Neurol., 92, 465.

HeYN, L. G. (1924). J. Amer. Med. Ass., 82, 1019.

Hollenhorst, R. W., and Henderson, J. W. (1951). Amer. J. med. Sci., 221, 211.

KeIL, H. (1940). Arch. intern. med., 66, 109.

KINNey, T. D., and MAHER, M. M. (1940). Amer. J. Path., 16, 561.

LeVER, W. F., and HAanes, M. (1954). A.M.A. Arch. Derm., 69, 123.

Lisman, J. V. (1947). Arch. Ophthal. (Chicago), 37, 155.

LoNDON, R. D. (1950). J. Pediat., 36, 817.

NutT, A. B. (1951). Proc. roy. Soc. Med., 44, 979. 
O'Leary, P. A., and Waisman, M. (1940). Arch. Derm. Syph. (Chicago), 41, 1001.

OPPEL, T. W., COKER, C., and MilHORAT, A. T. (1950). Ann. intern. Med., 32, 318.

PAGEL, W., WOOLF, A. L., and ASHER, R. (1949). J. Path. Bact., 61, 403.

SHEARD, C. (1951). Arch. intern. Med., 88, 640.

Simpson, J. R. (1953). Proc. roy. Sac. Med., 46, 288.

TALBOTT, J. H., and Moleres FERRANDIS, R. (1956). "Collagen Diseases". Grune and Stratton, New York.

WAGNER, E. L. (1863). Arch. Heilk. (Lpz.), 4, 282.

Walton, J. N., and AdAMS, R. D. (1958). "Polymyositis", p. 70. Livingstone, Edinburgh.

WedgwoOd, R. J. P., CoOK, C. D., and CoHEN, J. (1953). Pediatrics, 12, 447. 